\title{
ANALGESIC EFFICACY AND TOLERABILITY OF TRAMADOL VERSUS LOW DOSE TRAMADOL-PARACETAMOL IN PATIENTS WITH GASTRO INTESTINAL SURGERY
}

Monica Nepal, ${ }^{1}$ Rama Paudel, ${ }^{2}$ Rajesh Paudel, ${ }^{3}$ Narayan Gautam ${ }^{4}$

\begin{abstract}
INTRODUCTION

Analgesic regimens with an improved efficacy and tolerability balance have potential to improve acute pain management, and thus reduce the progression into chronic pain. Hence, an opportunity was gained to compare analgesic efficacy and tolerability of Tramadol (T) $1.5 \mathrm{mg} / \mathrm{kg}$ versus low dose Tramadol $1 \mathrm{mg} / \mathrm{kg}$-Paracetamol 1000mg (T-P) in patients with Gastro Intestinal (GI) surgery.
\end{abstract}

\section{MATERIAL AND METHODS}

The study was a hospital based prospective, observational study conducted in sixty post-operative GI surgery patients at Universal College of Medical Sciences-Teaching Hospital, Ranigaon, Bhairahawa, Nepal. One group received Tramadol $1.5 \mathrm{mg} / \mathrm{kg}(\mathrm{n}=30)$ while the other group received Tramadol $1 \mathrm{mg} / \mathrm{kg}$ with Paracetamol $1000 \mathrm{mg}(\mathrm{n}=30)$. The primary efficacy outcome measures were pain intensity difference (PID) and sum of pain intensity difference (SPID) whereas secondary efficacy measures included number of patients who require rescue medication, haemodynamic parameter, their quality of sleep in the night and satisfaction with their medication. For tolerability, adverse effect was noted that occurred during study time intervals.

\section{RESULTS}

Mean pain intensity differences assessed on Numerical Rating Scores (NRS) were significantly better for Group T-P compared to Group T at all the points except $0.5,1$ and $6 \mathrm{hrs}$. The sum of pain intensity difference over $8,16,24,48 \mathrm{hrs}$ for Group T-P was significantly superior to Group T. Two patients in Tramadol group required rescue medication. Satisfaction to the pain medication was comparatively higher for Tramadol-Paracetamol group.

\section{CONCLUSION}

Tramadol-Paracetamol had more pronounced analgesic effect with lower incidence of side effect than Tramadol alone. Thus, low dose Tramadol-Paracetamol is better option for management of post-operative pain in patient with GI surgery.

KEYWORDS Efficacy, Low dose Tramadol-Paracetamol, Pain Intensity Difference, Tolerability, Tramadol

1. Department of Pharmacy and Biomolecular Sciences, Universita Delgi Studi di Milano, Milan, Italy

2. School of Biomedical Sciences, Trinity Medical Sciences University, Ratho Mill, St. Vincent and the Grenadines

3. Department of Surgery, Universal College of Medical Sciences, Bhairahawa, Nepal

4. Department of Biochemistry, Universal College of Medical Sciences, Bhairahawa, Nepal

DOI: http//doi.org/10.3126/jucms.v7i2.27136

For Correspondence

Monica Nepal

Dept. of Pharmacy and Biomolecular Sciences

Universita Delgi Studi di Milano

Milan, Italy

E-mail: nepalmonica04@gmail.com 


\section{INTRODUCTION}

The definition of pain states that it is a subjective sensory and emotional experience hence its evaluation and the approach to treatment requires close collaboration with the patient. ${ }^{1,2}$ Postoperative pain is the most frequent type of pain seen by surgeons but it tends to be underestimated, inadequately treated and therefore not fully assessed with regard to its harmful effect on the patient and its impediment of a successful surgical outcome. ${ }^{3}$ Effective postoperative pain control is an essential component of the care of the surgical patient. ${ }^{4}$ Despite the availability of a wide variety of analgesic drugs, routine management of post-operative pain remains a continuing challenge.

Acetaminophen is a para-aminophenol, non-opiate, that works centrally possibly through inhibition of prostaglandin synthesis. ${ }^{7}$ Tramadol, a centrally acting analgesic, consists of two enantiomers, both of which contribute to analgesic activity via different mechanisms. (+) Tramadol and the metabolite $(+)$ O-Desmethyl-Tramadol (M1) are agonists of the $\mu$ opioid receptor. (+) Tramadol also stimulates presynaptic release of serotonin and inhibits serotonin reuptake whereas (-) tramadol inhibits norepinephrine reuptake. $^{8}$

Use of multiple analgesics, especially with different mechanisms of action and pharmacokinetics, can overcome the efficacy and safety limitations of the individual agents and can provide additive, or even synergistic, pain relief.,

The aim of this study is therefore to examine the efficacy and tolerability by assessing and monitoring adverse effect after intravenous injection of Tramadol versus low dose Tramadol Paracetamol since it is widely used in post-operative pain medication in GI surgery.

\section{MATERIAL AND METHODS}

The study was a hospital based prospective, observational study, conducted in sixty post-operative GI surgery patients who fulfilled inclusion criteria for the period of six months from July 2017 to December 2017 at Universal College of Medical Sciences-Teaching Hospital Ranigaon, Bhairahawa, Nepal. The study was approved by Institutional Ethical Committee with registration number (UCMS/IRC/079/17).

The exclusion criteria was set up for known hypersensitivity to Tramadol, Paracetamol, or non-steroidal anti-inflammatory medication, severe cardiopulmonary, renal or liver disease, morbid obesity, history of complications during and after surgery, intake of monoamine oxidase inhibitors within 30 days prior to surgery until 72 hours after the final visit.

A total of 60 patients were randomly divided into two groups, each comprising 30 patient's Group Tramadol and Group Tramadol plus Paracetamol. One group received Tramadol (Group T) $1.5 \mathrm{mg} / \mathrm{kg}$ thrice a day as IV bolus while the other group received Tramadol $1 \mathrm{mg} / \mathrm{kg}$ as IV bolus with Paracetamol $1000 \mathrm{mg}$ (Group T-P) as an IV infusion separately thrice a day immediately after surgery for 48 hours.

\section{Height weight and body mass index}

Height was measured using stadiometer. Weight was measured using weighing machine and Body Mass Index (BMI) was calculated by applying weight in $\mathrm{kg}$ divided by height in meter square.

\section{Pain assessment}

Pain was assessed at different time interval 0,30 minutes and $1,2,4,6,8,16,24,48$ hrs using numeric rating scale. Patients were asked to circle the number between 0 and 10 that fits best to their pain intensity. Zero usually represents 'no pain at all' whereas the upper limit represents 'the worst pain ever possible'. The pain level was assessed from the quality of sleep using 5 point scale ( 0 -no sleep, 1 -frequent waking, 2 -shallow sleep every night, 3-good sleep, 4-deep sleep) as was done by Paudel R et al (2017). ${ }^{11}$ At the end of $48 \mathrm{hrs}$, patients were asked to rank the quality of pain relief on a four point pain satisfaction scale with 1-Strongly dissatisfied, 2-Dissatisfied, 3-Satisfied, 4-Strongly satisfied.

\section{Efficacy criteria}

The primary efficacy outcome measures were pain intensity difference (PID) assessed at different time intervals (0-0.5, 0 $1,0-2,0-4,0-6,0-8,0-16,0-24,0-48)$ and sum of pain intensity difference (SPID) for the (0-8 hr, 0-16 hr, 0-24 hr, 0 $48 \mathrm{hr}$ ) whereas secondary efficacy measure included number of patients who require rescue medication, haemodynamic parameter, their quality of sleep in the night and satisfaction with their medication.

\section{Pain intensity difference (PID)}

Pain intensity difference is defined as the difference in pain intensity at various time points versus baseline. ${ }^{12}$

$$
P I D_{t}=P I_{\text {baseline }}-P I_{t}
$$

Where, $P I D_{\mathrm{t}}$ is the pain intensity difference score and $P I_{\mathrm{t}}$ pain intensity calculated at each post-baseline time point.

\section{Sum of pain intensity difference (SPID)}

It is calculated by the sum of the time-weighted pain intensity difference (difference between current pain and pain at baseline) multiplied by the interval between ratings. ${ }^{12}$ 


$$
\operatorname{SPID}_{t_{i}-t_{i+n}}=\sum_{t_{i}}^{t_{i+n}}\left(P I D_{i}\right) *\left(t_{i+1}-t_{i}\right)
$$

Where, $t_{i}$ is the scheduled assessment time, $t_{i+1}$ from the adjacent time point, $P I D_{\mathrm{i}}$ is the pain intensity difference score calculated each post-baseline time point.

\section{Haemodynamic parameter}

Blood pressure at different time interval was measured using sphygmomanometer and pulse and $\mathrm{SpO}_{2}$ was measured using pulse oximeter.

\section{Tolerability}

Adverse event like nausea and vomiting, dizziness, sedation, dry mouth, headache, dyspnoea, sweating, drowsiness and abdominal discomfort was observed between the groups.

\section{Statistical analysis}

Statistical analysis was done using Statistical Package for Social Science (SPSS) version 22, Inc., Chicago, IL, USA.Data were expressed in mean, standard deviation (SD) and percentage. Students t-test and chi square test were performed to determine the significance. $\mathrm{P}$ value $<0.05$ was considered to be statistically significant. The graphical representations were done with Prism Graph Pad version 7.03.

\section{RESULTS}

In present study $36(60 \%)$ patients were male out of which 14 (46.67\%) in Tramadol group and $22(73.33 \%)$ in TramadolParacetamol group, 24 (40\%) patients were female out of 16 $(53.3 \%)$ in Tramadol group and $8(26.7 \%)$ in TramadolParacetamol group. [Fig. 1a] The mean and standard deviation of demographic features like age, weight, height and BMI observed to be statistically non-significant difference $\mathrm{P}>0.05$ between two groups. [Fig. 1b] The age of patient in this study ranged from 15 to 75 years. Majority of them were in age group 26 to 35 years comprising four $(13.33 \%)$ in Tramadol group and 14 (46.66\%) in Tramadol-Paracetamol group.

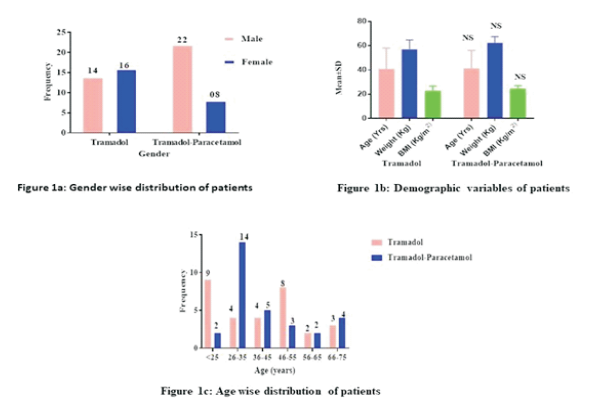

Figure 1. Bar diagram showing distribution of Demographic features of GI surgery patients
However, the maximum frequency of $9(30 \%)$ has been seen in $<25$ years in Tramadol group whereas 14 (46.67\%) in Tramadol-Paracetamol group respectively. [Fig. 1c]

The clinical diagnosis is statistically non-significant between Tramadol and Tramadol-Paracetamol $(\mathrm{p}=0.063)$ groups. [Table 1]

Table 1. Clinical diagnosis of the study subjects in two groups

\begin{tabular}{lcccc}
\hline \multicolumn{1}{c}{ Diagnosis } & \multicolumn{2}{c}{ Drug given } & Total & P value \\
& $\mathrm{T}(\mathrm{n}=30)$ & $\mathrm{T}-\mathrm{P}(\mathrm{n}=\mathbf{3 0})$ & & \\
\hline Appendectomy & $12(40 \%)$ & $13(43.33 \%)$ & $25(41.66 \%)$ & \\
Hernioplasty & $4(13.33 \%)$ & $3(10 \%)$ & $7(11.66 \%)$ & \\
Laprascopic Cholecystectomy & $12(40 \%)$ & $5(16.66 \%)$ & $17(28.33 \%)$ & 0.063 \\
Lapratomy & $2(6.66 \%)$ & $9(30 \%)$ & $11(18.33 \%)$ & \\
\hline
\end{tabular}

Mean SBP and DBP was observed to be statistically nonsignificant at any interval of time $\mathrm{p}>0.05$ between two groups. [Fig. 2a] Pulse rate were observed to be significantly differ at time interval of $4 \mathrm{hrs}, 6 \mathrm{hrs}, 8 \mathrm{hrs}, 16 \mathrm{hrs}, 24 \mathrm{hrs}$ and $48 \mathrm{hrs}$ $(p<0.001)$. However, Initial pulse rate at Baseline (BL) 30 min, $1 \mathrm{hr}$ and $2 \mathrm{hrs}$ are statistically non-significant difference. [Fig. 2b] $\mathrm{SpO}_{2}$ (\%) was statistically significantly difference at $4 \mathrm{hrs}$ but remains statistically non-significant at all intervals of time from BL, 0 hr to $48 \mathrm{hrs}(\mathrm{p}>0.05)$ in two groups. [Fig. 2c]

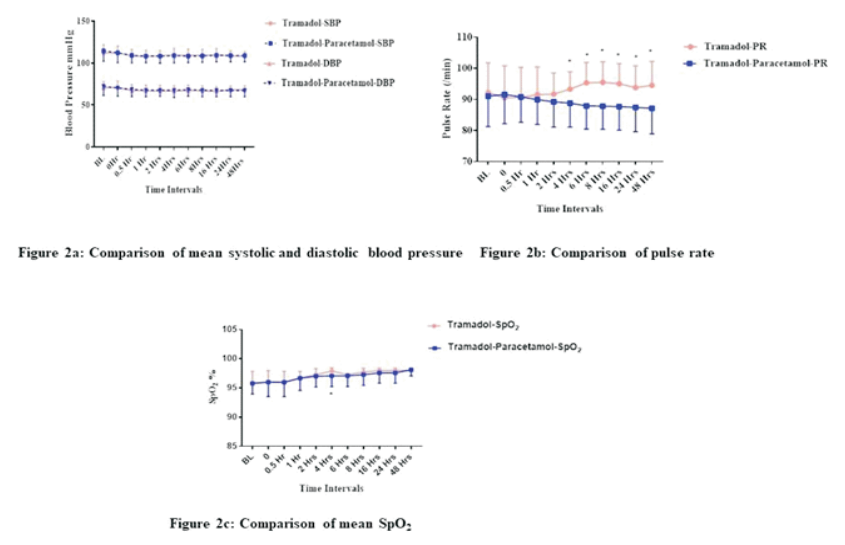

Figure 2. Mean \pm SD of hemodynamic parameter in GI surgery patients

Pain score at BL, $30 \mathrm{~min}$ and $1 \mathrm{hr}$ were statistically nonsignificant difference $(\mathrm{p}>0.05)$ between two groups. However, pain score there after interval of $2 \mathrm{hrs}-48 \mathrm{hrs}$ were observed to be significantly difference between two groups $(\mathrm{p}<0.05)$. [Fig. 3a] MPID at BL, $30 \mathrm{~min}, 1 \mathrm{hr}$ and $6 \mathrm{hrs}$ were statistically non-significant difference $(p>0.05)$ between two groups. However, MPID there after interval of $2 \mathrm{hrs}, 4 \mathrm{hrs}, 8$ hrs, $16 \mathrm{hrs}, 24 \mathrm{hrs}$ and $48 \mathrm{hrs}$ were observed to be significantly difference between two groups $(\mathrm{p}<0.05)$. [Fig.3b] The SPID 
of the two groups were taken from $8 \mathrm{hrs}$ to $48 \mathrm{hrs}$ with constant time interval of $8 \mathrm{hrs}$. The present study showed that, SPID interval of 0-8 hrs statistically non-significant while 0-16 hrs, 0-24 hrs and 0-48 hrs were observed to be statistically significant difference between two groups $(p<0.05)$. [Fig. 3c]
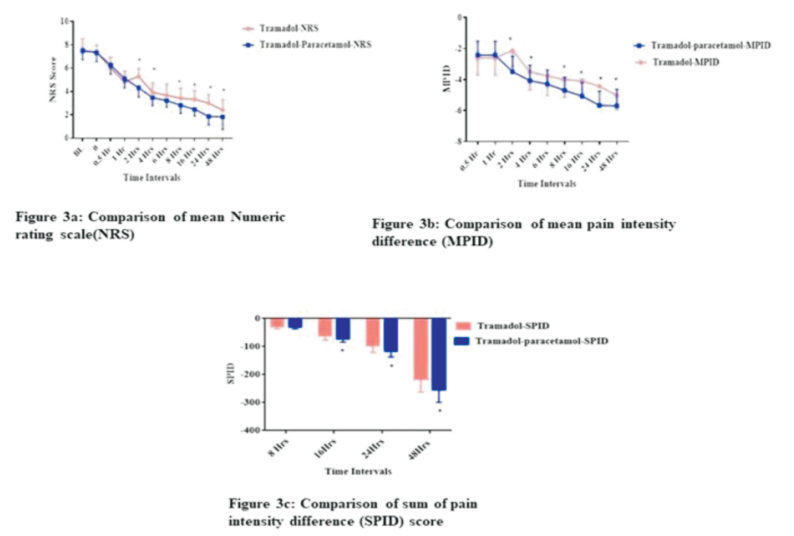

Figure 3. Mean \pm SD of various ways expressing pain intensity in GI surgery patients

Patient's satisfaction is statistically significant between Tramadol and Tramadol-Paracetamol $(\mathrm{p}=0.001)$ groups. [Table 2]

Table 2. Distribution of level of satisfaction with postoperative pain management in the study subjects in two groups

\begin{tabular}{ccccc}
\hline Patient's Satisfaction & \multicolumn{2}{c}{ Drugs given } & Total & P value \\
& $\mathrm{T}(\mathrm{n}=\mathbf{3 0})$ & $\mathrm{T}-\mathrm{P}(\mathrm{n}=\mathbf{3 0})$ & & \\
\hline Strongly dissatisfied & $7(23.33 \%)$ & $1(3.33 \%)$ & $8(13.33 \%)$ & \\
Dissatisfied & $7(23.33 \%)$ & $0(0 \%)$ & $7(11.66 \%)$ & \\
Satisfied & $14(46.66 \%)$ & $24(80 \%)$ & $38(63.33 \%)$ & $0.001^{*}$ \\
Strongly satisfied & $2(6.66 \%)$ & $5(16.67 \%)$ & $7(11.66 \%)$ & \\
\hline
\end{tabular}

Adverse drug effect is statistically non-significant between Tramadol and Tramadol-Paracetamol $(\mathrm{p}=0.180)$ groups. [Table 3 ]

Table 3. Distribution of Adverse Drug Effect (ADE) of study subjects in two groups

\begin{tabular}{lllll}
\hline \multicolumn{1}{c}{ ADE } & \multicolumn{2}{c}{ Drugs given } & Total & P-value \\
& \multicolumn{1}{c}{$\mathrm{T}(\mathrm{n}=30)$} & \multicolumn{1}{c}{ T-P $(\mathrm{n}=30)$} & $(\mathrm{n}=60)$ & \\
\hline Abdominal discomfort & $4(13.33 \%)$ & $1(3.33 \%)$ & $5(8.33 \%)$ & \\
Dizziness & $2(6.66 \%)$ & $0(0 \%)$ & $2(3.33 \%)$ & \\
Dry mouth & $4(13.33 \%)$ & $1(3.33 \%)$ & $5(8.33 \%)$ & \multirow{2}{*}{0.180} \\
Nausea/vomiting & $2(6.66 \%)$ & $0(0 \%)$ & $2(3.33 \%)$ & \\
No.ne & $1(3.33 \%)$ & $0(0 \%)$ & $1(1.66 \%)$ & \\
\hline
\end{tabular}

Rescue analgesia is statistically non-significant between Tramadol and Tramadol-Paracetamol $(\mathrm{p}=0.489)$ groups. [Table 4]

Table 4. Distribution of rescue analgesia of study subjects in two groups

\begin{tabular}{lllll}
\hline Rescue Analgesia & \multicolumn{2}{c}{ Drugs given } & Total & P value \\
& $\mathrm{T}(\mathrm{n}=30)$ & $\mathrm{T}-\mathrm{P}(\mathrm{n}=30)$ & & \\
\hline Morphine & $1(3.33 \%)$ & $0(0 \%)$ & $1(1.66 \%)$ & \\
Pethidine & $1(3.33 \%)$ & $0(0 \%)$ & $1(1.66 \%)$ & 0.489 \\
None & $28(93.33 \%)$ & $30(93.33 \%)$ & $58(96.66 \%)$ & \\
\hline
\end{tabular}

Sleep score is statistically non-significant between Tramadol and Tramadol-Paracetamol, in day $1(\mathrm{p}=0.619)$, in day 2 $(\mathrm{p}=0.253)$ respectively. [Table 5]

Table 5. Distribution of sleep score of study subjects in two groups

\begin{tabular}{lccc}
\hline Day & \multicolumn{2}{c}{ Drug given } & P value \\
& $\mathrm{T}(\mathrm{n}=30)$ & $\mathrm{T}-\mathrm{P}(\mathrm{n}=30)$ & \\
\hline 1 & $0.87 \pm 0.860$ & $0.77 \pm 0.63$ & 0.619 \\
2 & $1.53 \pm 0.692$ & $1.33 \pm 0.71$ & 0.253 \\
\hline
\end{tabular}

\section{DISCUSSION}

Although traditionally the mainstay of postoperative analgesia is opioid based, increasingly more evidence exists to support a multimodal approach with the intent to reduce opioid side effects and improve pain scores.

The male to female ratio was observed to be 3:2 with male predominance which is in congruence to the study done by Emir E et al. ${ }^{13}$ Mean weight and BMI was statistically nonsignificant difference in both groups showing drugs were delivered to the proportionate and effectiveness of drug dose delivery in each group.

Paracetamol combination with tramadol is uniquely effective because of the complimentary pharmacology and synergism; their combined analgesic effect is more than the sum of analgesic effects of both drugs, thus reducing side effects. Reducing the dose of tramadol to $1 \mathrm{mg} / \mathrm{kg}$ and combining it with Paracetamol $1 \mathrm{~g}$ orally decreased the incidence of side effects of tramadol without reducing analgesic efficacy. ${ }^{14}$ Oral medications cannot be tolerated or may experience compromised GI absorption function after surgery. Hence there is need for the parenteral formulations of the study medication. So injectable form of Tramadol and low dose Tramadol-Paracetamol can well tolerated systemically without GI dysfunction. 
In the present study, mean PID was significantly greater in intervals $2 \mathrm{hrs}$ to $48 \mathrm{hrs}$ in Tramadol-Paracetamol group as compared to Tramadol group in these time intervals. However, it was non-significant at 0.5 and $1 \mathrm{hr}$ in both groups. SPID is an outcome measure that summarises treatment response over a clinically relevant period. In Tramadol-Paracetamol group SPID observed at all these time was significantly greater except $0-8 \mathrm{hr}$. The combination of analgesics from different classes may provide additive analgesic effects with lesser side effects than when a single drug is used and also provide different mechanisms of action and therefore multimodal coverage of a wider spectrum of pain.

Rawal N conducted randomized, double-blind, doubledummy, multicenter trial demonstrated that postoperative pain following ambulatory hand surgery was effectively managed in the majority of patients by tramadol 37.5 $\mathrm{mg} /$ Paracetamol $325 \mathrm{mg}$ combination treatment with no statistically significant differences in efficacy to tramadol 50 mg monotherapy but with fewer AEs. In contrast to this study, Rawal $\mathrm{N}$ has shown the use of monotherapy and double therapy can be done according the feasibility and ease with patients management. ${ }^{15}$

James R. Fricke Jr. conducted randomized, double-blind, placebo-controlled trial in 456 patients demonstrated that Tramadol/Acetaminophen (APAP) $75 \mathrm{mg} / 650 \mathrm{mg}$ provides better analgesia than tramadol $100 \mathrm{mg}$ in the management of moderate-to-severe acute pain. Our study is in congruence to the study done Fricke JR showing Tramadol/APAP to be more effective to manage pain in oral surgery. ${ }^{16}$

In the study done by Ali S et al, the mean pain scores were comparable between the two groups at almost all time intervals. None of the patients had a score of more than 6 . More patients in group TP $(43 \%)$ than in group T $(27 \%)$ had a pain score more than 3 (VAS score 46), but this difference is statistically non-significant $(\mathrm{P}=0.08) .{ }^{17}$ However, in this study the pain score was high only at Baseline and $0 \mathrm{hr}$ while less than 7 pain score in all other time intervals which were statistically significant difference in all time intervals except at $6 \mathrm{hrs}$ between Tramadol and Tramadol-Paracetamol groups. The pharmacokinetics of the two drugs may vary for their plasma half-life showing non-significant at $6 \mathrm{hrs}$ but thereafter the difference in the pain score exist showing the fall in the drugs level in plasma however, the prolong efficacy in decline pain score was found in the Tramadol-Paracetamol group as compared to tramadol group in time intervals.

Fricke et al found that nausea, dizziness, and vomiting occurred more frequently in the Tramadol group than in the Tramadol-Paracetamol group. ${ }^{16}$ Kiliçaslan et al found no significant difference in $\square$ nausea and vomiting between the groups $(\mathrm{P}>0.05) .{ }^{18}$ Rawal $\mathrm{N}$ et al reported that TramadolParacetamol treatment showed a better safety profile than tramadol alone, and overall incidence of nausea, vomiting, and sedation scores was markedly lower in the combination group. ${ }^{15,19}$ In present study, more patients had side effects in tramadol group compared to Tramadol-Paracetamol group but the difference was statistically non-significant $(\mathrm{P}>0.05)$. Gastrointestinal and nervous system disorders were reported with a $15.4 \%$ and $11.4 \%$ lower frequency, respectively, for Tramadol-Paracetamol treatment. ${ }^{20}$

Tramadol-sparing did not lower the analgesic efficacy of the combination tablet, probably owing to the individual compounds acting additively. ${ }^{9}$ By contrast, Arici et $\mathrm{al}^{21}$ reported a $21 \%$ incidence of nausea and vomiting in their Paracetamol group and a $67 \%$ incidence in their placebo group. Arslan et $\mathrm{al}^{22}$ noted nausea incidences of $72 \%$ in their placebo group and $48 \%$ and $25 \%$ in their two paracetamol groups and vomiting incidences of $50 \%$ in their placebo group and $27 \%$ and $12 \%$ in the corresponding Paracetamol groups.

In our study, intraoperative pain was assessed by haemodynamic responses. physiological changes often accompany pain, for example sympathetic response resulting in increase in HR and BP, but studies indicate that these physiological and endocrine events occurring concurrently with pain may be a general response to stress and not unique to pain. $^{23}$ Although Tramadol has minimal cardiovascular affects, it has been reported to cause a slight but clinically insignificant increase in HR and blood pressure. ${ }^{13}$ On the other hand, Paracetamol does not affect the cardiovascular system at therapeutic doses. ${ }^{24}$

Similar to our study, the study done by Emir E et al found no significant differences between the two groups in terms of systolic, diastolic and MBP, and HR at any time-point. The decrease in MBP and HR values did not exceed $20 \%$ and no additional treatment was deemed necessary in any subject. ${ }^{13}$ Tramadol has been reported to provide adequate analgesia without causing changes in the respiratory rate, $\mathrm{SpO}_{2}$ and blood gases, or causing hypoxia, and is thought to have a clear advantage over opioids. ${ }^{25}$

A relationship between sleep and pain has long been recognized, but mostly in the context of chronic pain where determination of causal relationships is problematic because it is difficult to disentangle effects of poor sleep on pain from the effects of pain on sleep. In the present study, both the groups showed improvement in quality of sleep during the study period.

The key to adequate pain control is to reassess the patient and determine if he or she is satisfied with the outcome.In the present study, 7 strongly dissatisfied, 7 dissatisfied,14 
satisfied, 2 strongly satisfied in Tramadol group and 1 strongly dissatisfied, 0 dissatisfied, 24 satisfied , 5 strongly satisfied in Tramadol-Paracetamolgroup $(p=0.001)$. By contrast Emir E et al in Group T, the satisfaction score was rated as good by four $(13.3 \%)$ patients and as very good by $26(86.7 \%)$ patients. In Group $\mathrm{P}$, the satisfaction score was rated as good by two $(6.7 \%)$ patients and as very good by $28(93.3 \%)$ patients. $^{13}$

\section{CONCLUSION}

The result of the study demonstrated that low dose TramadolParacetamol increased the effectiveness of analgesics administered in the postoperative period. This combination reduced the incidence of side effects and provided effective analgesia. The combined Tramadol-Paracetamol regimen was superior to Tramadol alone in reducing the intensity of pain during the treatment.

\section{ACKNOWLEDGEMENTS}

We would like to acknowledge all the patients, staffs and faculties of Department of Surgery for providing us space to carry out this study.

\section{REFERENCES}

1 Ossipov MH, Dussor GO, Porreca F. Central modulation of pain. JCli Invest. 2010; 120(11):3779-87.

2 Swieboda P, Filip R, Prystupa A, Drozd M. Assessment of pain: types, mechanism and treatment. Ann Agric Environ Med. 2013 ;1(12):2-7.

3 Colletti V, Carner M, Vincenzi A, Dallari S, Mira E, Benazzo M, Cosentino G, Bellussi L, Passali D. Intramuscular tramadol versus ketorolac in the treatment of pain following nasal surgery: a controlled multicenter trial. CurrTher Res. 1998; 59(9):608-18.

4 Chou R, Gordon DB, de Leon-Casasola OA, Rosenberg JM, Bickler S, Brennan T, et al. Management of Postoperative Pain: A Clinical Practice Guideline From the American Pain Society, the American Society of Regional Anesthesia and Pain Medicine, and the American Society of Anesthesiologists' Committee on Regional Anesthesia, Executive Commi. J Pain. 2016;17(2) :131-57.

5 Pareek A, Chandurkar N, Gupta A, Desai Y, Kumar S H, Swamy A, et al. Comparative evaluation of efficacy and safety of etodolac and diclofenac sodium injection in patients with postoperative orthopedic pain. Curr Med Res Opin. 2011;27 (11):2107-15.

6 Mohammed S,Manjula BP, Sunil BV.Comparative study of intravenous paracetamol and intravenous tramadol for postoperative analgesia in laparotomies. Anesth Essays Res. 2015;9(3):314-9.

7 Farquhar-Smith P, Gubbay A. Tramadol and acetaminophen combination for chronic non-cancer pain. Expert Opin Pharmacother. 2013;14(16):2297-304.
8 Keskinbora K, Aydinli I. An atypical opioid analgesic: Tramadol. Agri. 2006;18(1):5-19.

9 Desmeules J, Rollason V, Piguet V, Dayer P. Clinical pharmacology and rationale of analgesic combinations. Eur J Anaesthesiol Suppl. 2003;20(Suppl 28):7-12.

10 Ali M, Khan FA. Comparison of analgesic effect of tramadol alone and a combination of tramadol and paracetamol in daycare laparoscopic surgery. Eur J Anaesthesiol.2009;26(6):475-9.

11 Paudel R, Deka A,Gupta HK,Nepal HP.Comparative evaluation of analgesic efficacy of tramadol and diclofenac-sodium in postoperative orthopedic patients. Int J Basic Clin Pharmacol. 2017 ;6 (3) :643-7.

12 Johnson JR. Standard Methods for Analysis and Reporting of VAS or NRS Derived Pain Relief Response Scores. 2016;1-22.

13 Emir E, Serin S, Erbay H, Sungurtekin H, Tomatir E. Tramadol Versus Low Dose Tramadol-paracetamol for Patient Controlled Analgesia During Spinal Vertebral Surgery. Kaohsiung J Med Sci. 2010;26(6):308-15.

14 Ali M, Khan FA. Comparison of analgesic effect of tramadol alone and a combination of tramadol and paracetamol in daycare laparoscopic surgery. Eur J Anaesthesiol.2009;26(6):475-9.

15 Rawal N, Macquaire V, Catalá E, Berti M, Costa R, Wietlisbach M. Tramadol/paracetamol combination tablet for postoperative pain following ambulatory hand surgery: A double-blind, double-dummy, randomized, parallel-group trial. J Pain Res. 2011 ; 8(4):103-10.

16 Fricke JR, Hewitt DJ, Jordan DM, Fisher A, Rosenthal NR. A double-blind placebo-controlled comparison of tramadol acetaminophen and tramadol in patients with postoperative dental pain. Pain. 2004;109(3):250-7

17 Ali S, Sofi K, Dar AQ. Comparison of Intravenous Infusion of Tramadol Alone with Combination of Tramadol and Paracetamol for Postoperative Pain after Major Abdominal Surgery in Children. Anesth Essays Res. 2017;11(2):472-476

18 Kilicaslan A, Tuncer S, Yuceaktas A, Uyar M, Reisli R. The effects of intravenous paracetamol on postoperative analgesia and tramadol consumption in cesarean operations. Agri Derg. 2010;22(1):7-12.

19 Alfano G, Grieco M, Forino A, Meglio G, Pace MC, Iannotti M. Analgesia with paracetamol/tramadol vs. paracetamol/codeine in one day-surgery: A randomized open study. Eur Rev Med Pharmacol Sci. 2011;15(2):205-10.

20 Scott LJ, Perry CM. Tramadol: a review of its use in perioperative pain. Drugs. 2000;60(1):139-76.

21 Arici S, preemptive anglegesic effects of intravenous paracetamol in total abdominal hysterectomy. J Turkish Soc Algol. 2009; 21(2):54-61.

22 Arslan M, Celep B, Cicek R, Kalender HO, Yilmaz H. Comparing the efficancy of preemptive intravenous paracetamol 
on the reducing effect of opioid usage in cholecystectomy, J Res Med Sci. Mar;18(3):172-7.

23 Christensen P, Brandt MR, Rem J, Kehlet H. Influence of extradural morphine on the adrenocortical and hyperglycaemic response to surgery. Br J Anaesth. 1982;54(1):23-7.

24 Avellaneda C, Gómez-Luque A, Martos F, Rubio M, Sarmiento J, De La Cuesta FS. The effect of a single intravenous dose of metamizol $2 \mathrm{~g}$, ketorolac $30 \mathrm{mg}$ and propacetamol $1 \mathrm{~g}$ on haemodynamic parameters and postoperative pain after heart surgery. Eur J Anaesthesiol. 2000;17(2):85-90.

25 Duthie DJR. Remifentanil and tramadol. Br J of Anaesth. 1998; 81:51-57. 